\title{
Lapatinib-loaded nanocolloidal polymeric micelles for the efficient treatment of breast cancer
}

\author{
Gunjan Vasant Bonde ${ }^{1,2}$, Gufran Ajmal ${ }^{1,3}$, Sarita Kumari Yadav ${ }^{1,4}$, Pooja Mittal ${ }^{1,5}$, Brahmeshwar Mishra ${ }^{1 *}$ \\ ${ }^{1}$ Department of Pharmaceutical Engineering Technology, Indian Institute of Technology (Banaras Hindu University), Varanasi, India. \\ ${ }^{2}$ School of Health Sciences, University of Petroleum and Energy Studies, Dehradun, India. \\ ${ }^{3}$ Bharat Institute of Technology, Meerut, India. \\ ${ }^{4}$ Department of Pharmacy, Moti Lal Nehru Medical College, Allahabad, India. \\ ${ }^{5}$ Department of Pharmaceutics, Maharishi Markandeshwar University, Ambala, India.
}

\section{ARTICLE INFO \\ Received on: 30/04/2020 \\ Accepted on: 15/06/2020 \\ Available online: 05/09/2020}

\section{Key words:}

Anticancer agent, nanocarrier, passive targeting, polymeric micelles, SKBr3.

\begin{abstract}
This study aims at preparing and evaluating lapatinib-loaded polymeric micelles for the better treatment of breast cancer (BC). LP-loaded polymeric micelles (LP-PMs) were prepared as per our previous studies by using Soluplus ${ }^{\circledR}$ as the polymer. Therefore, we employed the lyophilization technique using mannitol as a cryoprotectant and further conducted in vitro and in vivo anticancer efficacy studies, in addition to our previously reported works. We found that the lyophilized LP-PMs were sufficiently stable and retained encapsulated drugs. Furthermore, their smooth surface was visualized on the atomic force microscopy. The X-ray powder diffractogram of LP-PMs showed successful encapsulation of Lapatinib; however, the presence of few drug molecules on the surface was evidenced by energy dispersive X-ray analysis. Furthermore, LP-PMs showed sustained release of drugs, with selective drug release in an acidic environment, resembling that of a tumor. The LP-PMs exhibited higher cytotoxicity against SKBr3 BC cells and also induced effective inhibition of the growth of the tumor in vivo when compared to that of lapatinib solution and marketed formulation. The results of this study indicate the greater potential of LP-PMs for the efficient treatment for $\mathrm{BC}$.
\end{abstract}

\section{INTRODUCTION}

Breast cancer $(\mathrm{BC})$, standing on the second rank for mortality, is predicted to cause about 2.4 million cases by 2025 among women (WHO, 2018). Human epidermal growth factor receptor 2 (HER2) expressing BC constitutes about $30 \%$ instances among the types of BC (Wei et al., 2015).

Lapatinib (LP) is an anticancer drug that possesses specificity for HER2-positive BC. Additionally, LP is a dual inhibitor of Epidermal growth factor receptor (EGFR) and HER2 (Xia et al., 2002); however, its clinical use is limited due to its higher plasma protein binding (about 99\%), high hydrophobicity, and poor aqueous solubility. Hence, LP is administered frequently,

"Corresponding Author

Brahmeshwar Mishra, Department of Pharmaceutical Engineering Technology, Indian Institute of Technology (Banaras Hindu University), Varanasi, India.E-mail: bmishrabhu@ rediffmail.com causing less patient compliance, high side effects, and demands a higher cost for treatment (Bonde et al., 2018). Therefore, it is important to employ the benefits of nanocolloidal drug delivery systems to enhance the therapeutic value and patient compliance of LP. Up until now, the only commercially available dosage form for LP is Tykerb ${ }^{\circledR}$ (oral tablets by GlaxoSmithKline).

In the past few years, nanocolloidal systems, like Polymeric micelles (PMs), have been shown to be the most attractive nanocarriers for solubility and bioavailability improvement of hydrophobic drugs, owing to their unique coreshell structure (Bonde et al., 2018; Chiappetta and Sosnik, 2007; Guo et al., 2016). The structure of PMs consists of a hydrophobic core, while the hydrophilic shell results in their higher stability (Dehghan Kelishady et al., 2015). Additionally, the particle size of PMs $(<200 \mathrm{~nm})$ is also favorable for their accumulation in tumors by an enhanced permeation and retention (EPR) effect.

Furthermore, the amphiphilic polymers, like Soluplus ${ }^{\circledR}$, an Food and Drug Administration (FDA) approved triblock 
polymer, can form PMs by self-assembly (Kou et al., 2018). Soluplus $^{\circledR}$, at a concentration exceeding the critical micelle concentration, tends to self-assemble instantly with nanocolloidal micelles in an aqueous environment, thereby decreasing interaction with the aqueous environment and achieving thermodynamic stability by reducing free energy (Alvarez-Rivera et al., 2016). Scientists have demonstrated the high potential of Soluplus ${ }^{\circledR}$ micelles for the improvement of aqueous solubility (Varela-Garcia et al., 2018) and sustained release of poorly soluble drugs (Xia et al., 2016; Yu et al., 2013; Zeng et al., 2017).

Considering these issues, we had optimized and prepared LP-loaded polymeric micelles (LP-PMs) in our previous study, reporting only in vitro evaluations (Bonde et al., 2020). The in vitro evaluations indicated that LP-PMs possessed the potential for higher encapsulation efficiency (EE) and drug loading (DL) of this highly hydrophobic drug, LP. With the sustained release of LP, LP-PMs were also shown to be hemocompatible. The LP-PMs were sufficiently stable at $5^{\circ} \mathrm{C} \pm 3^{\circ} \mathrm{C}$ in the solution phase, with an estimated shelf-life of 12 months, which was further prolonged to 15 months by lyophilization. However, their stability was greatly compromised within three months of stability testing at room temperature. Therefore, an attempt was made to improve the stability of LP-PMs at room temperature.

In view of this perspective, in this research, we attempted lyophilization of optimized LP-PMs as per our previous report. Moreover, the physicochemical characterizations and in vitro releases of newly prepared lyophilized LP-PMs were carried out to evaluate the effect of lyophilization on the evaluation parameters. We furthered our research, in continuation to our previous reports, to evaluate in vitro cytotoxicity studies in SKBr3 HER2-positive $\mathrm{BC}$ cell line using the 3-(4,5-dimethylthiazol-2-yl)-2,5-diphenyl tetrazolium bromide (MTT) assay and in vivo anticancer efficacy studies in a tumor-bearing nude mice cancer model.

\section{MATERIALS AND METHODS}

Pluronics $^{\circledR}$ F127 (PF127) and Soluplus ${ }^{\circledR}$ were kindly supplied by BASF India Limited (Navi Mumbai, India). Lapatinib was procured from Xi'an Kerui Biotechnology Co. Ltd (Xi'an, China). BC cell line (SKBr3) was purchased from the National Centre for Cell Science, Pune.

\section{Preparation of micelles}

The thin-film hydration method was employed for the preparation of LP-PMs, as reported earlier (Bonde et al., 2020). For lyophilization, mannitol was used as a cryoprotectant. An appropriate quantity of mannitol was added to the dispersion of LP-PMs. The obtained dispersion was then allowed to freeze. The frozen dispersion was then lyophilized and stored at room temperature.

\section{Solid-state characterization of LP-PMs}

\section{Surface morphology}

Atomic force microscopy (Atomic force microscopy (AFM), NT-MDT, NTGRA PRIMA, Russia) was utilized to observe the surface roughness of spin-coated LP-PMs. The instrument was run at the scanning rate of $0.5 \mathrm{~Hz}$ in a semi-contact scanning mode.

\section{Energy dispersive $X$-ray analysis (EDX)}

Elemental compositions of LP-PMs were analyzed on the EDX instrument (Ametek, NJ). The dense region of the micelles was focused on using X-ray radiation at a fixed angle to determine the elements present in the micelle.

$X$-ray powder diffraction (XRPD) study

To determine changes in the crystalline structure and surface composition of excipients and the LP, before and after fabrication, XRPD patterns of LP, excipients, physical mixture, and LP-PMs were recorded using Rigaku's portable X-ray diffractometer (Rigaku, Japan).

\section{Entrapment efficiency and Drug Loading}

LP-PMs were evaluated for EE and DL using the UVspectrophotometric (Shimadzu UV-1800) method. The samples were appropriately diluted with methanol (see supplementary material for detailed procedure) and the absorbance was read at $261.5 \mathrm{~nm}$. The amount of LP was determined from the standard curve of LP in methanol, and EE and DL were calculated.

\section{Stability studies}

The lyophilized LP-PMs were stored at different environmental conditions for 6 months, as shown in Table 1. At the intervals of 0, 3, and 6 months their EE was evaluated. For stability studies, International Council for Harmonization (ICH) guidelines (Q1A (R2)) were followed and Minitab ${ }^{\circledR}$ ver. 17 was used to estimate the shelf life (Mittal et al., 2019).

\section{In vitro drug release}

LP-PMs were assessed for drug release by modifying the previously reported dialysis bag method (Wei et al., 2015). Briefly, a presoaked dialysis membrane bag (molecular cut-off weight of $14 \mathrm{kDa}$, Fisherbrand) was filled with $1 \mathrm{ml}$ of optimized LP-PMs (equivalent to $2 \mathrm{mg}$ of LP) and the two ends were sealed. The dialysis bag was then introduced into $50 \mathrm{ml}$ of the release medium, phosphate buffered saline (PBS: $\mathrm{pH} 7.4$ and PBS: $\mathrm{pH}$ 5.0), and was maintained at $37^{\circ} \mathrm{C} \pm 0.5^{\circ} \mathrm{C}$ with 100 Revolutions per minute (RPM) stirring speed. The aliquot was withdrawn at appropriate time intervals and replenished with an equal volume of the fresh released medium. The sink condition was maintained by adding $0.5 \%$ Tween 80 to ensure drug release. The released LP was quantified by the UV-spectrophotometer as previously described. The cumulative percentage release was also calculated.

Table 1. Results of evaluations before and after stability studies.

\begin{tabular}{ccc}
\hline & \multicolumn{2}{c}{ Results of evaluations $^{\mathrm{a}}$} \\
\hline Conditions & $\mathbf{3 0}^{\circ} \mathbf{C} \pm \mathbf{2}^{\circ} \mathbf{C} / \mathbf{6 5} \% \mathbf{R H} \pm \mathbf{5 \%} \mathbf{~ R H}$ & $\mathbf{5}^{\circ} \mathbf{C} \pm \mathbf{3}^{\circ} \mathrm{C}$ \\
Time (month) & $\mathbf{E E ~ ( \% ) ~}$ & $\mathbf{E E ~ ( \% ) ~}$ \\
0 & $85.22 \pm 8.57$ & $85.22 \pm 8.57$ \\
3 & $83.59 \pm 7.80$ & $84.13 \pm 8.57$ \\
6 & $81.77 \pm 8.40$ & $82.86 \pm 8.15$ \\
\hline
\end{tabular}

aAll the results are mentioned as mean \pm standard deviation, $\mathrm{n}=3$. 


\section{In vitro screening of anticancer efficacy}

\section{Cell culture}

McCoy's 5A medium was enriched with $10 \%$ fetal bovine serum, $100 \mathrm{U} / \mathrm{ml}$ penicillin, and $100 \mathrm{mg} / \mathrm{ml}$ streptomycin, and was used for culturing $\mathrm{SKBr} 3 \mathrm{BC}$ cell line. They were incubated at $37^{\circ} \mathrm{C}$ in the presence of $5 \% \mathrm{CO}_{2}$. For in vitro screening, the cells were seeded in 96-well culture plates at a density of $2 \times 10^{4}$ cells/ well and cultured for 24 hours after trypsinization (Bonde et al., 2020, Jeong et al., 2015).

\section{Cytotoxicity assay}

The cytotoxicity of LP-PMs was assessed by the MTT assay. Next day, the cells were treated with appropriate dilutions (ranging from 0.05 to $100 \mu \mathrm{g} / \mathrm{ml}$ ) of LP-PMs and lapatinib solution (LS) and incubated. On the following day, $100 \mu \mathrm{l}$ MTT solution $(0.5 \mathrm{mg} / \mathrm{ml})$ was added to the wells after removing culture media and was incubated for further 4 hours. The MTT solution was then replaced with $100 \mu 1$ Dimethyl sulfoxide (DMSO) to solubilize formazan crystals formed by the living cells only. The optical density at $590 \mathrm{~nm}$ of each well was then recorded using a microplate reader. The obtained data were analyzed to calculate $\mathrm{IC}_{50}$ values and percentages of cell viability by using GraphPad Prism software by nonlinear regression (Wan et al., 2015a; Wei et al., 2015).

Cell viability was calculated as follows:

$$
\text { Cell viability } \%=\left(A_{\text {test }} / A_{\text {control }}\right) \times 100
$$

\section{In vivo screening of anticancer efficacy}

$\mathrm{Balb} / \mathrm{c}$ nude mice were housed in individually ventilated cages and fed with a standard sterilized diet and sterilized water ad libitum. Animals were maintained in 12-12-hour light-dark cycles and $25^{\circ} \mathrm{C}$ air-conditioned, controlled noise rooms. Balb/c nude mice of $8-10$ weeks were used for the experiment. The xenograft Balb/c nude mice models were developed as previously discussed in the literature (Wang et al., 2014) and experimental procedures were carried out in accordance with the protocol approved (vide approval no. Dean/2018/CAEC/639) by Central Animal Ethical Committee, Banaras Hindu University, Varanasi (India), following guidelines of the committee for the purpose of control and supervision of experiments on animals and principles of laboratory animal care (National Institute of Health). Briefly, SKBr3 cells were harvested and injected subcutaneously to the nude mice after resuspending cells in PBS. The tumor volume was measured and calculated using the following formula:

$$
V=\left(W^{2} \times L\right) / 2
$$

where $\mathrm{V}=$ volume of tumor; $\mathrm{W}=$ shortest diameter; and $\mathrm{L}=$ longest diameter.

On the formation of tumors of approximately 700-800 $\mathrm{mm}^{3}$ in size, different treatment groups ( $\mathrm{n}=6$ per group) were formed by randomly dividing the mice. Different mice groups were injected intravenously in the tail vein and received the following treatment: Group 1: PBS (control); Group 2: LS $(10 \mathrm{mg} / \mathrm{kg} / 3$ days); Group 3: LP-PMs (10 mg/kg/3 days); Group 4: Tykerb®, a marketed tablet in dosage form of LP (oral administration $100 \mathrm{mg}$ / $\mathrm{kg} / 1$ day). On day 28 , the mice were euthanized and tumors were immediately harvested and their volume was noted.

\section{RESULTS AND DISCUSSIONS}

\section{Preparation of micelles}

Lyophilization was employed to convert the liquid dosage to a solid dosage. The dispersion of LP-PMs was transformed into a solid, fluffy, white powder form. It was stored at room temperature. All the evaluations were carried out using the lyophilized LP-PMs.

\section{Solid-state characterization of LP-PMs}

\section{Surface morphology}

AFM images revealed smooth surfaces with spherical shapes of micelles. Figure 1A shows an average peak-to-peak height of $60 \mathrm{~nm}$, which may be correlated to the apparent diameter of micelle. However, the size visualized by AFM was smaller than that found by dynamic laser scattering (DLS) in our previous studies. The observed difference was attributed to the drying of PMs while spincoating the sample preparation for AFM (Chen et al., 2016). Nanodelivery systems, having a size below $200 \mathrm{~nm}$, generally facilitate the passive targeting of tumors by filtering nanocarriers from the blood stream and restraining them to tumors by the EPR effect (Huo et al., 2015). Thus, the prepared LP-PMs were anticipated to have a better tumor-targeting capability as their size is well below 100 $\mathrm{nm}$. Their spherical shape and smooth surface, characterized by AFM, may facilitate their transit through the blood stream and can minimize opsonization and adsorption of the proteins.

\section{Energy dispersive $X$-ray analysis}

The optimized micelles were subjected to EDX analysis to verify the elements present in the micelle. As shown from Figure 1B, the EDX spectra of LP-PMs showed the presence of carbon, hydrogen, and oxygen with fluorine and sulfur that are typical constituents of LP and absent in Soluplus ${ }^{\circledR}$ and PF127 molecules. The results not only indicated the successful encapsulation of drugs inside the micelles, but some of the drugs were also present on the surface of the LP-PMs.

\section{$X$-ray powder diffraction study}

LP, excipients, physical mixture, LP-PMs, and lyophilized LP-PMs were subjected to XRPD analysis to determine their physical states and to verify changes in the crystallinity of the drug; the recorded X-ray diffractograms are shown in Figure 1C. The sharp peaks at $18.04^{\circ}, 18.9^{\circ}$, and $21.02^{\circ}$ in the LP diffractograms indicated its crystalline structure; the data resembled that reported in previous literature (Wan et al., 2015b). Soluplus ${ }^{\circledR}$ exhibited a broad halo with no sharp peaks revealing its amorphous nature (Zhang et al., 2016), whereas PF127 had two sharp peaks at $19.22^{\circ}$ and $23.36^{\circ}$. The diffractogram of the physical mixture showed the presence of all the peaks corresponding to LP, Soluplus ${ }^{\circledR}$, and PF127; however, the peak intensity was quite reduced. LP-PMs showed peaks relating to Soluplus ${ }^{\circledR}$ and PF127 only, but no characteristic peak of LP was observed, which confirmed the encapsulation of drugs in the core of LP-PMs, and Soluplus ${ }^{\circledR}$ and PF127 formed the sheath of LPPMs, as expected (Bonde et al., 2020). Furthermore, lyophilized LP-PMs constituted peaks of mannitol with the addition of PF127 only. Lyophilized LP-PMs showed rather mere additions of some 
peaks of mannitol (used as a cryoprotectant) in the diffractogram of LP-PMs. In conclusion, the presence of fluorine and sulfur in the EDX spectra and the disappearance of the peaks of LP in the XRPD formulation confirmed successful incorporation of LP in an amorphous form in LP-PMs.

\section{Entrapment efficiency and Drug loading}

LP-PMs entrapped LP to a higher extent as EE and DL were found to be $85.23 \% \pm 8.57 \%$ and $2.48 \% \pm 0.25 \%$, respectively. As previously reported, micelles have a core-shell structure where

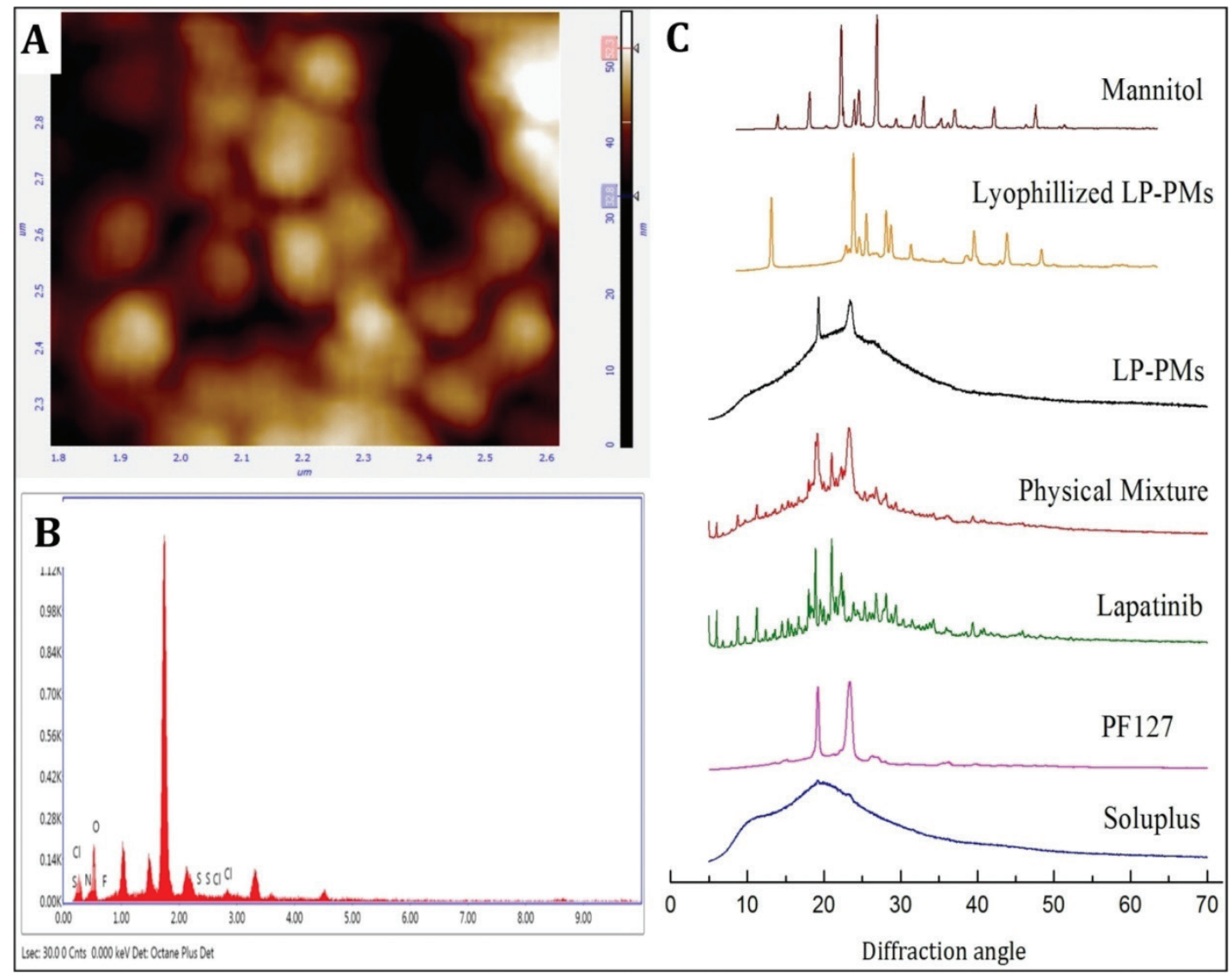

Figure 1. (A) The atomic force microscopic image showing the morphology of LP-PMs; (B) photograph illustrating the presence of elements analyzed by energy dispersive X-ray studies; (C) X-ray powder diffractogram of LP, excipients, their physical mixture, and LP-PMs before and after lyophilization.

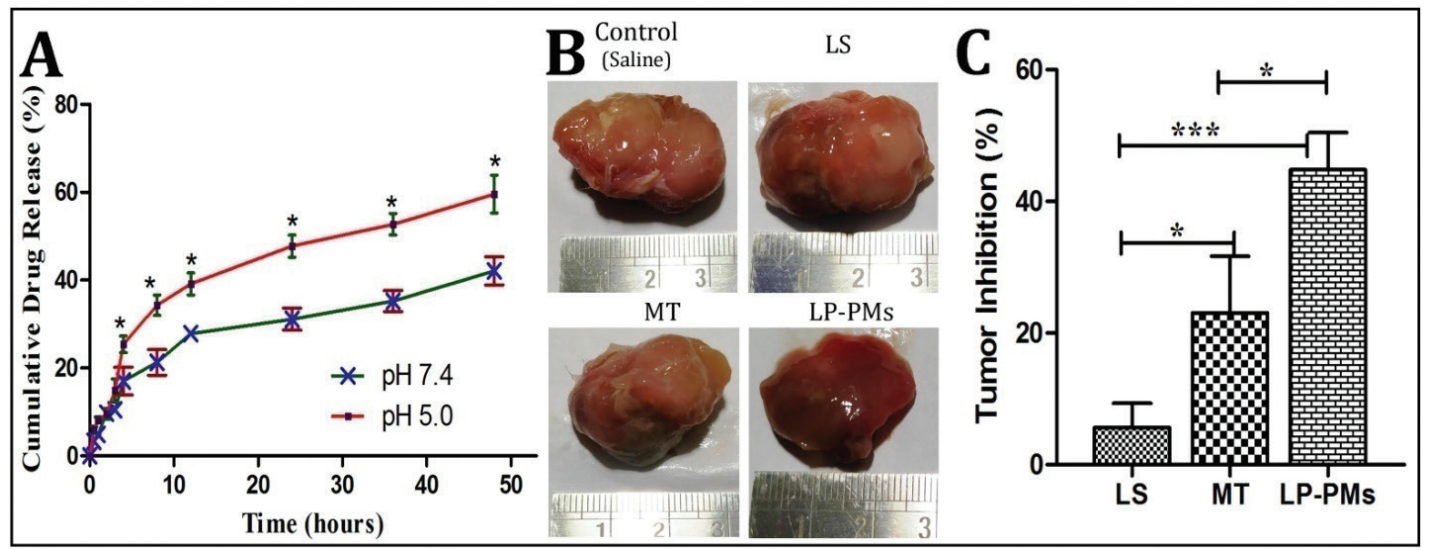

Figure 2. (A) Drug release profile of LP-PMs in media of different $\mathrm{pH}$; (B) In vivo anticancer efficacy showing tumor photographs and $(C)$ percentage inhibition of tumor growth post-treatment. 
the hydrophobic drugs find the appropriate space in the core. Encapsulation of LP in the hydrophobic core of PMs can explain the observed high EE and can serve as an insoluble drug reservoir; the findings are in good agreement with the previous reports (You et al., 2007). High entrapment and loading efficiency ensure more availability of the drug around the target, thereby alleviating the systemic side effects.

\section{In vitro drug release}

Figure 2A shows the drug release profile of LP-PMs over the course of 48 hours in two different $\mathrm{pH}$ conditions. The drug release from the LP-PMs followed a biphasic pattern irrespective of $\mathrm{pH}$, consisting of the first phase of faster drug release, lasting for almost 12 hours, followed by a phase of sustained release. In $\mathrm{pH}$ 5.0, the total LP release was found to be $49 \%$ and $64 \%$ at the end of 24 and 48 hours, respectively, while nearly $31 \%$ of LP had been

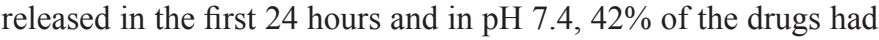
been released in 48 hours. Furthermore, the drug release data were tested to different kinetic models and found that LP release fits with the Higuchi model. The value of Peppas' exponent " $n$ " was found to be 0.416 and 0.381 at $\mathrm{pH} 7.4$ and $\mathrm{pH} 5.0$, respectively.

In this study, we had conducted in vitro drug release studies in two release media that simulated the biological environment resembling the blood stream $(\mathrm{pH} 7.4)$ and tumor ( $\mathrm{pH}$ 5.0). These conditions had served to predict the drug release from LP-PMs in different biological sites where they were expected to transit or accumulate. As seen from Figure 2A, drug release followed a biphasic pattern. Within the first 24 hours, a comparatively higher release of LP was observed in both $\mathrm{pH}$, which seemed to be due to the dissolution/solubilization of LP present in bulk of LP-PMs dispersion and bound to the external surfaces of LP-PMs to a minimal extent. The phase was then followed by a reduction in the total amount of LP release and achieved sustained release behavior, as expected. A significantly higher cumulative drug release was observed at $\mathrm{pH} 5.0$ compared to that at $\mathrm{pH}$ 7.4. Furthermore, different kinetic models were verified for the obtained drug release data and found that LP release fits with the Higuchi model, indicating that diffusion was the principal mechanism of drug release. The value of Peppas' exponent " $n$ " was found to be 0.381 for $\mathrm{pH} 5.0$ and 0.416 for $\mathrm{pH} 7.4$, indicating that LP release followed Fick's diffusion.

LP-PMs were fabricated and were expected to safely and specifically deliver the drug to the tumor. The intention was assumed to be fulfilled as LP-PMs showed a higher drug release at $\mathrm{pH} 5.0$ that simulated the tumor environment. On the other hand, a lower drug release at $\mathrm{pH} 7.4$ resembling blood compartments ensures comparatively a lesser loss of drugs during their transit through blood and may minimize the dose-related toxicities to the other organs of the body. The LP-PMs exhibited hydrogen bonding between LP and Soluplus ${ }^{\circledR}$. In an acidic environment, hydrogen bonding might weaken due to the presence of an excess of available hydrogen atoms, resulting in a reduction in partial negative charge on oxygen atoms. The effect might have dissociated the hydrogen bonding between LP and Soluplus ${ }^{\circledR}$ in an acidic environment. Due to the weakening or dissociation of hydrogen bonding to some extent in the acidic environment, the rigidity of micelles had been reduced. It might have resulted in the observed higher drug release at pH 5.0 than in $\mathrm{pH} 7.4$ (Bonde et al., 2020). Additionally, a sustained release of drugs from the LP-PMs was witnessed and was credited to the micellar association of amphiphiles which rigorously maintain the integrity against their dissociation and their resistivity to mere dilutions by body fluids (Batrakova et al., 2006; Lombardo et al., 2015). In conclusion, poor water-soluble drugs, like LP, can be formulated as nanocolloidal micelles; in this case, LP-PMs can serve as a sustained release dosage form for tumor-specific delivery of LP.

\section{Stability studies}

We observed the effect of different storage conditions on $\mathrm{EE}$, indicating the loss of drugs due to environmental conditions. The changes observed in EE were nonsignificant $(p<0.05)$ when LP-PMs were stored in both the conditions as given in Table 1. The results corroborated that the lyophilization resulted in the transformation of liquid dispersion to a solid powder form that markedly enhanced its stability, especially at $30^{\circ} \mathrm{C} \pm 2{ }^{\circ} \mathrm{C} / 65 \%$ Relative humidity $(\mathrm{RH}) \pm 5 \% \mathrm{RH}$, as compared to our previous studies, where LP-PMs in the dispersion form were aggregated within 3 months. Therefore, the lyophilized LP-PMs were stored at room temperature and served our purpose.

\section{In vitro screening of anticancer efficacy}

\section{Cell culture}

For this study, SKBr3 was selected, in particular, for in vitro evaluations. SKBr3 BC cell line is characterized by HER2 overexpression (Greenshields et al., 2019) and can serve as best for this study.

\section{Cytotoxicity assay}

The observed $\mathrm{IC}_{50}$ values of LP-PMs and LS were about 1.60 and $8.188 / \mathrm{ml}$, respectively. The results revealed a better anticancer efficacy of LP-PMs than free drugs in both the cell lines.

Lapatinib exhibited antagonist activity for EGFR and HER2 and acted by binding reversibly to intracellular Adenosine triphosphate (ATP)-binding site of their kinase domain and prevented autophosphorylation, resulting in growth arrest and/ or apoptosis in tumor cell lines that express EGFR and HER2 receptors. The MTT-based assay was carried out on SKBr3 BC cells to evaluate the anticancer efficacy of LP-PMs. The cell cytotoxicity is measured in terms of $\mathrm{IC}_{50}$ values; the concentration of drugs required for the inhibition of cell growth by $50 \%$ was compared with the controls.

Findings of the MTT assay suggested that LP-PMs inhibits the HER2 expressing cell lines as $\mathrm{IC}_{50}$ values for $\mathrm{SKBr} 3$ were significantly lower than that treated with LS. Additionally, LP-PMs exhibited almost six-fold lower $\mathrm{IC}_{50}$ values against SKBr3 compared to that of LS, indicating more significant cytotoxic effects of LP-PMs. Therefore, micellar drug delivery of LP, indeed, raised its cytotoxicity to cancer cells, and the results are concurrent with previous reports (Wan et al., 2015b).

\section{In vivo screening of anti-cancer efficacy}

Followed by in vitro characterization of LP-PMs, we shifted further for their evaluation of in vivo antitumor efficacy by employing the ectopic xenograft mice model bearing in-situ SKBr3 tumors. After 28 days of treatment, the tumors were excised and 
the volumes were measured; the results are shown in Figure 2B. A significant reduction in growth rate was observed in the group treated with LP-PMs when compared to the control group, while the control tumors continued to grow at a faster rate. The dramatic reduction in the tumor growth rate was observed after treatment by marketed formulation and LP-PMs. The inhibition of tumor growth by $5.66 \% \pm 3.64 \%$ was observed posttreatment by LS, whereas $23.09 \% \pm 8.56 \%$ inhibition was produced by treatment with the marketed oral formulation. In the case of the animal group treated with LP-PMs, $44.83 \% \pm 5.64 \%$ inhibition was observed, which was significantly different than that produced in animal groups treated with LS and marketed formulation (Figure 2C).

The enhanced antitumor effects are attributed to the nanosize, i.e., below $100 \mathrm{~nm}$, of the LP-PMs, which is a preamble for the EPR effects of nanocarriers enabling their higher tumor accumulation. Another reason might be the encapsulation of the drug inside the core of PMs that might have reduced the loss of drug due to plasma albumin-binding during circulation and made it available at tumor site via the EPR effect. It is worth mentioning that the dose of LP-PMs was 10 times lesser than marketed oral tablets. Therefore, LP-PMs might prove to be an effective anti-tumor treatment, with LP-PMs as a much lesser dose. The observed fact, indeed, confirmed the possibility of dose reduction to a great extent without compromising the anticancer efficacy by the change of route of administration, as well as employing nanocolloidal micellar drug delivery system for such a high hydrophobic drug. Overall, LP-PMs serve as a booster for the therapeutic potential of LP.

\section{CONCLUSION}

The clinical use of LP is limited due to its low solubility, high dose, and high protein binding. To address such issues, we employed a nanocolloidal micellar drug delivery approach and prepared LP-PMs using Soluplus ${ }^{\circledR}$ as an amphiphilic polymer. The lyophilization converted the dispersion phase to a solid powder of LP-PMs, thereby enhancing the stability of formulation at room temperature, unlike the observed aggregation in LP-PMs dispersion at room temperature. The LP-PMs had a smooth surface, spherical shape, and a size less than $100 \mathrm{~nm}$, which suggested their passive targeting potential by the EPR effect. The LP was successfully encapsulated, except few traces, on the outer surface of the micelles. Furthermore, LP-PMs were able to provide a sustained release with selectivity in drug release in an acidic $\mathrm{pH}$, mimicking the tumor environment. Moreover, the enhanced cytotoxicity of LP-PMs against SKBr3 BC is attributed to higher encapsulation and solubility of LP. In addition, effective inhibition of the growth rate of tumors in the in vivo mice model confirmed the anticancer efficacy of LP-PMs and of lapatinib solution and marketed formulation. In conclusion, the findings of in vitro and in vivo evaluations suggest that micellar drug delivery of LP can serve as an efficient treatment option for BC.

\section{CONFLICT OF INTEREST}

The authors declare no conflict of interest.

\section{FUNDING}

None.

\section{REFERENCES}

Alvarez-Rivera F, Fernández-Villanueva D, Concheiro A, Alvarez-Lorenzo C. $\alpha$-lipoic acid in soluplus ${ }^{\circledR}$ polymeric nanomicelles for ocular treatment of diabetes-associated corneal diseases. J Pharm Sci, 2016; 105(9):2855-63.

Batrakova EV, Bronich TK, Vetro JA, Kabanov AV. Polymer micelles as drug carriers. In: Torchilin VP, (ed.). Nanoparticulates as drug carriers. World Scientific, Singapore, pp 57-93, 2006.

Bonde GV, Ajmal G, Yadav SK, Mittal P, Singh J, Bakde BV, Mishra B. Assessing the viability of Soluplus ${ }^{\circledR}$ self-assembled nanocolloids for sustained delivery of highly hydrophobic lapatinib (anticancer agent): Optimisation and in-vitro characterisation. Colloids Surf B Biointerfaces, 2020; 185:110611.

Bonde GV, Yadav SK, Chauhan S, Mittal P, Ajmal G, Thokala S, Mishra B. Lapatinib nano-delivery systems: a promising future for breast cancer treatment. Expert Opin Drug Del, 2018; 15(5):495-507.

Chen G, Jaskula-Sztul R, Harrison A, Dammalapati A, Xu W, Cheng Y, Gong S. KE108-conjugated unimolecular micelles loaded with a novel HDAC inhibitor thailandepsin-A for targeted neuroendocrine cancer therapy. Biomaterials, 2016; 97:22-33.

Chiappetta DA, Sosnik A. Poly (ethylene oxide)-poly (propylene oxide) block copolymer micelles as drug delivery agents: improved hydrosolubility, stability and bioavailability of drugs. Eur J Pharm Biopharm, 2007; 66(3):303-17.

Dehghan Kelishady P, Saadat E, Ravar F, Akbari H, Dorkoosh F. Pluronic F127 polymeric micelles for co-delivery of paclitaxel and lapatinib against metastatic breast cancer: preparation, optimization and in vitro evaluation. Pharm Dev Tech, 2015; 20(8):1009-17.

Greenshields AL, Fernando W, Hoskin DW. The anti-malarial drug artesunate causes cell cycle arrest and apoptosis of triple-negative MDA-MB-468 and HER2-enriched SK-BR-3 breast cancer cells. Exp Mol Pathol, 2019; 107:10-22.

Guo C, Li M, Qi X, Lin G, Cui F, Li F, Wu X. Intranasal delivery of nanomicelle curcumin promotes corneal epithelial wound healing in streptozotocin-induced diabetic mice. Sci Rep, 2016; 6:29753.

Huo ZJ, Wang SJ, Wang ZQ, Zuo WS, Liu P, Pang B, Liu K. Novel nanosystem to enhance the antitumor activity of lapatinib in breast cancer treatment: therapeutic efficacy evaluation. Cancer Sci, 2015; 106(10):1429-37.

Jeong EG, Yoo HJ, Song B, Kim HP, Han SW, Kim TY, Cho DID. Evaluation of lapatinib powder-entrapped biodegradable polymeric microstructures fabricated by X-ray lithography for a targeted and sustained drug delivery system. Materials, 2015; 8(2):519-34.

Kou L, Sun R, Bhutia YD, Yao Q, Chen R. Emerging advances in P-glycoprotein inhibitory nanomaterials for drug delivery. Expert Opin Drug Del, 2018; 15(9):869-79.

Lombardo D, Kiselev MA, Magazù S, Calandra P. Amphiphiles self-assembly: basic concepts and future perspectives of supramolecular approaches. Adv Cond Matter Phys, 2015; 2015:22.

Mittal P, Vardhan H, Ajmal G, Bonde GV, Kapoor R, Mittal A, Mishra B. Formulation, optimization, hemocompatibility and pharmacokinetic evaluation of PLGA nanoparticles containing paclitaxel. Drug Dev Ind Pharm, 2019; 45(3):365-78.

Varela-Garcia A, Concheiro A, Alvarez-Lorenzo C. Soluplus micelles for acyclovir ocular delivery: formulation and cornea and sclera permeability. Int J Pharm, 2018; 552(1-2):39-47.

Wan X, Zheng X, Pang X, Zhang Z, Jing T, Xu W, Zhang Q. The potential use of lapatinib-loaded human serum albumin nanoparticles in the treatment of triple-negative breast cancer. Int J Pharm, 2015a; 484(1):1628.

Wan X, Zheng X, Pang X, Zhang Z, Zhang Q. Incorporation of lapatinib into human serum albumin nanoparticles with enhanced antitumor effects in HER2-positive breast cancer. Colloids Surf B Biointerfaces, 2015b; 136:817-27. 
Wang H, Li F, Du C, Wang H, Mahato RI, Huang Y. Doxorubicin and lapatinib combination nanomedicine for treating resistant breast cancer. Mol Pharm, 2014; 11(8):2600-11.

Wei Y, Xu S, Wang F, Zou A, Zhang S, Xiong Y, Jiang X. A novel combined micellar system of lapatinib and paclitaxel with enhanced antineoplastic effect against human epidermal growth factor receptor-2 positive breast tumor in vitro. J Pharm Sci, 2015; 104(1):165-77.

WHO. 2018. Cancer fact sheet. [ONLINE] Available via https:// www.who.int/en/news-room/fact-sheets/detail/cancer (Accessed 31 May 2019).

Xia D, Yu H, Tao J, Zeng J, Zhu Q, Zhu C, Gan Y. Supersaturated polymeric micelles for oral cyclosporine a delivery: the role of soluplussodium dodecyl sulfate complex. Colloids Surf B Biointerfaces, 2016; 141:301-10.

Xia W, Mullin R J, Keith BR, Liu LH, Ma H, Rusnak DW, Spector NL. Anti-tumor activity of GW572016: a dual tyrosine kinase inhibitor blocks EGF activation of EGFR/erbB2 and downstream Erk1/2 and AKT pathways. Oncogene, 2002; 21(41):6255.

You J, Hu FQ, Du YZ, Yuan H, Ye BF. High cytotoxicity and resistant-cell reversal of novel paclitaxel loaded micelles by enhancing the molecular-target delivery of the drug. Nanotechnology, 2007; 18(49):495101.
Yu H, Xia D, Zhu Q, Zhu C, Chen D, Gan Y. Supersaturated polymeric micelles for oral cyclosporine a delivery. Eur J Pharm Biopharm, 2013; 85(3):1325-36.

Zeng Y, Li S, Liu C, Gong T, Sun X, Fu Y, Zhang Z. Soluplus micelles for improving the oral bioavailability of scopoletin and their hypouricemic effect in vivo. Acta Pharmacol Sin, 2017; 38(3):424-33.

Zhang Y, Liu Y, Luo Y, Yao Q, Zhong Y, Tian B, Tang X. Extruded Soluplus/SIM as an oral delivery system: characterization, interactions, in vitro and in vivo evaluations. Drug Deliv, 2016; 23(6):1902-11.

\section{How to cite this article:}

Bonde GV, Ajmal G, Yadav SK, Mittal P, Mishra B. Lapatinib-loaded nanocolloidal polymeric micelles for the efficient treatment of breast cancer. J Appl Pharm Sci, 2020; 10(09):023-029. 